\title{
Accurate transaxial region-of-interest reconstruction in helical CT?
}

\author{
Rolf Clackdoyle, Frédéric Noo, Fabien Momey, Laurent Desbat, Simon Rit
}

\begin{abstract}
In conventional helical computed tomography (CT), the field-of-view is a cylinder centered on the axis of the helix. Here, we consider the situation where all measurement lines are blocked except those intersecting a small cylindrical region-ofinterest (ROI) not necessarily centered on the axis of the system. We address the question of image reconstruction inside the ROI. The patient boundary is assumed known, and we avoid the "interior problem" by assuming that the ROI includes part of the patient boundary. By applying analytic image reconstruction theory, we show that the entire cylindrical ROI can be reconstructed provided the pitch of the helix does not violate the well-known Tam-Danielsson detector condition. Using an iterative algorithm, we performed ROI reconstruction from simulated phantom data and from real patient data, and compared the results with fullfield reconstructions. Visually, the ROI reconstructed images perfectly matched the full-field reconstructions. However, there were small quantitative discrepancies near the interior boundaries of the ROIs, which we attribute to the known reduced stability at one side of the inverse truncated Hilbert transform. In conclusion, we have demonstrated mathematically that accurate transverse ROI reconstruction is possible for helical CT, although care must be taken near the interior boundary to achieve quantitative accuracy.
\end{abstract}

\section{INTRODUCTION}

Helical CT scanners with tens or hundreds of detector rows have been the standard for about 10 years. Image reconstruction theory has been well studied for this geometry: the main theoretical result is that as long as the helix pitch does not exceed the axial extent of the detectors, then mathematically exact image reconstruction is possible in principle. Region-of-interest (ROI) reconstruction refers to techniques for reconstructing a sub-volume from a reduced dataset. Our motivation in looking at this problem is the potential for dramatically reduced radiation dose that should be achievable. For reduced dose to be meaningful, the reconstruction quality inside the ROI should be comparable to the situation for fullfield scanning. In this work, we examine the theoretical issues behind ROI reconstruction in helical CT. We do not attempt to quantify the dose reduction here.

Image reconstruction theory for helical cone-beam scanning was the subject of intense research activity around

This work was partially supported by grant ANR-12-BS01-0018 (project "DROITE") from the Agence Nationale de la Recherche.

R. Clackdoyle, L. Desbat, are with the TIMC-IMAG laboratory (CNRS UMR 5525), Grenoble, France; e-mail: rolf.clackdoyle@univ-grenoblealpes.fr

F. Noo is with the Utah Center for Advanced Imaging Technologies, University of Utah, Salt Lake City, USA.

F. Momey was with the TIMC-IMAG laboratory, Grenoble, France and is now with the Hubert Curien laboratory (CNRS UMR5516), Saint Etienne, France.

S. Rit is with the CREATIS laboratory (CNRS UMR 5220), Lyon, France. the turn of the century [1]-[7]. The new challenges were the unavoidable axial truncation of the projections and the axially translating field-of-view which implied a simultaneous collection of partially-overlapping reconstruction problems. Chord-based image reconstruction methods arose from these developments, and resulted in a simple paradigm that clearly established the theoretical foundation for image reconstruction from helical scanning with a minimum detector (sometimes referred to as "minimal data"). Zou and Pan [8] provided this key development. Further developments such as efficient use of redundant detector information and variations on the helical trajectory continue to be explored, but we are only concerned here with conventional helical scanning and the question of data sufficiency for robust and reliable reconstruction.

ROI reconstruction with transaxial truncation for classical two-dimensional (parallel-beam and fanbeam) tomography was developed in the early 2000s and is now largely understood [9]. Chord methods play a central role in ROI reconstruction theory [10], [11] because reconstruction along a chord can be achieved provided the chord is visible in all projections along the sub-trajectory of the x-ray source that connect the endpoints of the chord. For helical cone-beam scanning, the benefits of chord reconstruction for ROI reconstruction with transaxial truncation were recognized early on [8],[12]-[14]. However, there were two difficulties. One difficulty, common to the two-dimensional case, was that chord reconstruction could only take place along visible chords (i.e., chords that were not truncated in the projections), so the ROI had to be geometrically expressible as the intersection of the object with a union of chords. The second difficulty was that, unlike the two-dimensional case, even if the ROI was contained in a union of line segments that crossed the entire object, there was no assurance that the chords (line segments with endpoints on the x-ray trajectory) were available, and usually they were not. For the helix, ROI reconstruction was only possible along the available chords. This point was discussed by Pack et al [12] who went much further and showed how reconstructions could occur along line segments that defined the ROI, provided the line segment intersected the trajectory (once) and provided a set of suitable chords intersected all points along these line segments. This approach allowed much more flexibility for ROI reconstruction with transaxial truncation in helical CT. However, the first difficulty remained, that the ROI had to be expressed as a union of line segments that crossed the object. In the two-dimensional case, this difficulty was removed in 2006 with a new theoretical result [15] on inversion of the truncated Hilbert transform whose consequence was that the line segments of the ROI only had to hit the boundary of 
the object on one side, not necessarily on both sides. This condition now admits any convex region that intersects the boundary of the object as a valid ROI, for the two-dimensional case. The corresponding analysis does not appear to have been carried over to helical scanning. To our knowledge, transverse truncation in helical scanning for ROIs that do not cross the object has not been treated in the literature.

The purpose of this work is to demonstrate that mathematically correct image reconstruction is possible in helical CT for a cylindrical ROI which intersects the boundary of the patient support which we assume to be an elliptical cylinder of known dimensions. No explicit analytic reconstruction method will be proposed here. Our contribution is a straight-forward and selfcontained demonstration that image reconstruction is possible in principle; in other words, that the helical ROI geometry provides sufficient data for mathematically correct image reconstruction. This fact is important for iterative reconstruction methods that, in general, blindly optimize a regularized cost function to produce an image that best matches the data. The results presented here suggest that such reconstructed images can be determined largely by the projection data rather than by the regularization term in the ideal situation of noiseless or low-noise data. (Helical ROI reconstruction is "data-driven").

In section 2, we review the image reconstruction principles that will be used, and in passing, we indicate how chord-based reconstruction theory easily establishes that helical reconstruction is possible for the long object problem. In section 3, we point out that using chords alone, it does not seem to be possible to resolve the helical ROI problem, but that the Mline concept introduced by [12] can be successfully applied. Section 4 presents some example ROI reconstructions from a helical CT geometry for both simulated phantom data and real patient projection data. The last section, section 5, consists of discussion and conclusions.

\section{REVIEW OF ROI RECONSTRUCTION PRINCIPLES}

\section{A. Cone-beam projection and backprojection}

We let $f$ denote the unknown three-dimensional (3D) density function. We assume that $f$ is known to be zero outside the infinite elliptical cylinder $E_{a, b}=\left\{x \in \mathbb{R}^{3} \mid\left(x_{1} / a\right)^{2}+\right.$ $\left.\left(x_{2} / b\right)^{2} \leq 1\right\}$ where $0<b \leq a$. The $\mathrm{x}$-ray source travels along a continuous trajectory which we parametrize using the scalar $\lambda \in\left[\lambda_{o}, \lambda_{e}\right]$. The source position is geometrically at the vertex of the cone-beam projection and denoted $\Phi(\lambda)$, and we simplify expressions such as $\Phi\left(\lambda_{A}\right)$ to $\Phi_{A}$. Later, we will explicitly define the helical trajectory but for now we only assume that the trajectory does not intersect $E_{a, b}$. Cone-beam projections are denoted with $g$ :

$$
g(\lambda, \theta)=\int_{0}^{\infty} f(\Phi(\lambda)+t \theta) d t
$$

where $\theta \in D_{\lambda} \subset S^{2}$ is a unit vector specifying the direction of a ray emanating from $\Phi(\lambda)$. The subset $D_{\lambda}$ of possible measured rays depends on the geometry of the detector with respect to the source at position $\Phi(\lambda)$. Now for $\left[\lambda_{1}, \lambda_{2}\right] \subset$ $\left[\lambda_{o}, \lambda_{e}\right]$ we define the differentiated backprojection (DBP) $b_{1,2}$ as the image obtained by the cone-beam backprojection of the derivative of the projections:

$$
b_{1,2}(x)=\left.\frac{1}{\pi} \int_{\lambda_{1}}^{\lambda_{2}} \frac{1}{\|x-\Phi(\lambda)\|} \frac{\partial}{\partial \lambda} g(\lambda, \theta)\right|_{\theta=\frac{x-\Phi(\lambda)}{\|x-\Phi(\lambda)\|}} d \lambda .
$$

\section{B. Hilbert transforms and inverses}

For the Hilbert transform in the direction $\alpha \in S^{2}$, operating on an image $f$, we use the notation $H_{\alpha} f$ defined by

$$
H_{\alpha} f(x)=\int_{-\infty}^{\infty} \frac{f(x-s \alpha)}{\pi s} d s
$$

and it is easily verified that $H_{-\alpha} f=-H_{\alpha} f$. Furthermore, the definition extends naturally to non-unit vectors: for any positive scalar $k, H_{k \alpha}=H_{\alpha}$. Note that $H_{\alpha} f(x)$ is a onedimensional convolution of the Hilbert kernel $1 / \pi s$ along the line $L_{x, \alpha}$ passing through $x$ in the direction $\alpha$. The Fourier transform of $1 / \pi s$ is $-i \operatorname{sgn}(\sigma)$ so it is immediately clear that $H_{\alpha} f$ can be inverted to obtain $f$ using the formula

$$
f(x)=-\int_{-\infty}^{\infty} \frac{H_{\alpha} f(x-h \alpha)}{\pi h} d h
$$

but it is important to note that the limits of integration cannot be reduced here because the integrand is non-zero everywhere.

ROI reconstruction using DBP techniques relies heavily on the capability to compute $f(x)$ from a truncated (or "finite") range of values of $H_{\alpha} f(x)$. Fixing $x$ inside the (known) support of $f$ and $\alpha \in S^{2}$, we consider the line segment defined as the intersection of line $L_{x, \alpha}$ with the support of $f$. We thus identify $s_{1}$ and $s_{2}$ such that $f(x+s \alpha)=0$ for all $s \notin\left(s_{1}, s_{2}\right)$, and we note in passing that from this definition, we have $0 \in\left[s_{1}, s_{2}\right]$. We assume that the values of $H_{\alpha} f(x+h \alpha)$ are available only for $h$ in the known interval $\left[h_{1}, h_{2}\right]$, and we want to obtain $f(x)$ from the available values of $H_{\alpha} f(x+h \alpha)$. We recall three important cases here, which will all be germane to the discussions in the next sections.

Case one: $\left[s_{1}, s_{2}\right] \subset\left(h_{1}, h_{2}\right)$. The most favorable situation is when there is "more of $H_{\alpha} f$ known than the unknown part of $f(x)$ " and an explicit formula, sometimes called the "twosided finite Hilbert inverse" can be applied. The formula can be found in [16] for example, and has the following form:

$$
\begin{aligned}
f(x)= & \frac{-1}{\sqrt{-h_{1} h_{2}}} \int_{h_{1}}^{h_{2}} \sqrt{\left(h-h_{1}\right)\left(h_{2}-h\right)} \frac{H_{\alpha} f(x-h \alpha)}{\pi h} d h \\
& +C
\end{aligned}
$$

noting that $0 \in\left[s_{1}, s_{2}\right] \subset\left(h_{1}, h_{2}\right)$ implies that $h_{1}<0$ and $h_{2}>0$, so $-h_{1} h_{2}>0$. The constant $C$ is independent of where point $x$ is taken along the line $L_{x, \theta}$ and can be determined in various ways (see [10], [11]), one of which uses the expression

$$
C=\frac{1}{\pi \sqrt{-h_{1} h_{2}}} \int_{s_{1}}^{s_{2}} f(x+s \theta) d s .
$$

which is available from the cone-beam measurements, so $C=$ $\left(1 / \pi \sqrt{-h_{1} h_{2}}\right) g\left(\lambda_{0}, \theta\right)$, assuming that the line $L_{x, \theta}$ intersects the trajectory at $\Phi\left(\lambda_{0}\right)$. The essential fact from case one is 
that there is a formula that can be used to invert the two-sided finite Hilbert transform.

Case two is the opposite extreme: $\left[h_{1}, h_{2}\right] \subset\left(s_{1}, s_{2}\right)$. Here we have that "less of $H_{\alpha} f$ is known than the unknown part of $f(x)$." For this situation, knowledge of $H_{\alpha} f(x+h \alpha)$ for $h \in\left[h_{1}, h_{2}\right]$ does not uniquely determine $f(x)$. There simply isn't enough information available.

Case three is the intermediate case $h_{1}<s_{1}<h_{2}<s_{2}$ (and an equivalent case of $s_{1}<h_{1}<s_{2}<h_{2}$ which is handled similarly). In this case there is an overlap of the regions of known $H_{\alpha} f$ and unknown $f(x)$. The result is something midway between those of cases one and two. It is known that for all $s \in\left(s_{1}, h_{2}\right), f(x+s \alpha)$ can be uniquely determined from $H_{\alpha} f(x+h \alpha)$ with $h \in\left[h_{1}, h_{2}\right]$. More importantly, $f(x+s \alpha)$ can be stably recovered from the $H_{\alpha} f$ values, although the stability constant becomes poorer as $s$ tends to $h_{2}$ [8],[17-19]. However, there is currently no known formula to explicitly invert this "one-sided finite Hilbert transform." For the purposes of image reconstruction, we consider that $f(x)$ can be reliably obtained from $H_{\alpha} f$ if $h_{1}<s_{1}<h_{2}$ and provided $h_{2}>0$. In practice, this inversion would need to be performed using a numerical scheme such as a POCS method described in [15], [20].

\section{Chord reconstruction and M-line reconstruction}

A central result in image reconstruction theory is the link between equations (2), and (3). Taking (1) and substituting into (2) yields the following formula

$$
b_{1,2}(x)=H_{\theta_{2}} f(x)-H_{\theta_{1}} f(x)
$$

where $\theta_{1}=\left(x-\Phi_{1}\right) /\left\|x-\Phi_{1}\right\|$ and $\theta_{2}=\left(x-\Phi_{2}\right) /\left\|x-\Phi_{2}\right\|$ are the direction vectors from the source locations $\Phi_{1}$ and $\Phi_{2}$ to $x$ respectively. This result has appeared in the literature in a slightly different form in [12], and a 2D version of equation (7) appeared in [9]. A simple demonstration is included in the appendix. Note that the cone-beam projections $g(\lambda, \cdot)$ for $\lambda \in\left[\lambda_{1}, \lambda_{2}\right]$ can be truncated, as long as the projection of (a small neighborhood of) $x$ is not truncated in any of these projections.

The right hand side of equation (7), the sum of two Hilbert transforms along different lines, is not invertible, and cannot be used to solve for $f(x)$. However, this equation is the foundation of the chord and M-line methods. Both methods are based on the observation that if $x$ lies on the straight line segment $\left[\Phi_{1}, \Phi_{2}\right]$ connecting $\Phi_{1}$ to $\Phi_{2}$, then $\theta_{2}=-\theta_{1}$. Recalling that $H_{-\theta_{1}}=-H_{\theta_{1}}$, we obtain from equation (7) the central equation of the chord method:

$$
b_{1,2}(x)=2 H_{\theta_{1,2}} f(x) \quad \text { where } x \in\left[\Phi_{1}, \Phi_{2}\right]
$$

with $\theta_{1,2}$ defined as $\theta_{1,2}=\left(\Phi_{1}-\Phi_{2}\right) /\left\|\Phi_{1}-\Phi_{2}\right\|$, and because of the position of $x$, we have $\theta_{1,2}=\theta_{2}=-\theta_{1}$.

The M-line method [12] is based on equation (10) below, which is also easily derived from equation (7). Again, $x$ is required to lie on some segment $\left[\Phi_{1}, \Phi_{2}\right]$. For any $\lambda_{M} \in$ $\left(\lambda_{o}, \lambda_{e}\right)$, we note that

$$
\begin{aligned}
b_{1, M}(x)+b_{2, M}(x)= & H_{\theta_{M}} f(x)-H_{\theta_{1}} f(x)+ \\
& H_{\theta_{M}} f(x)-H_{\theta_{2}} f(x) \\
= & 2 H_{\theta_{M}} f(x)
\end{aligned}
$$

where $\theta_{M}=\left(x-\Phi_{M}\right) /\left\|x-\Phi_{M}\right\|$ is the direction of the line, called the M-line, from $\Phi_{M}$ passing through $x$. The expressions $b_{1, M}(x)$ and $b_{2, M}(x)$ refer to the same backprojection expression given by equation (2), except with integration limits $\left(\lambda_{1}, \lambda_{M}\right)$ and $\left(\lambda_{2}, \lambda_{M}\right)$ respectively. Using the notation $b_{1,2, M}$ to refer to $b_{1, M}+b_{2, M}$ we express the M-line equation as

$$
b_{1,2, M}(x)=2 H_{\theta_{M}} f(x) \quad \text { where } x \in\left[\Phi_{1}, \Phi_{2}\right]
$$

Note that $\lambda_{M}$ is not necessarily larger than $\lambda_{2}$ or even than $\lambda_{1}$. However, the usual situation is $\lambda_{M} \in\left(\lambda_{1}, \lambda_{2}\right)$, including for our application of the M-line theory in section III.C.

Chord Reconstruction. A chord of the source trajectory is a straight-line segment $\left[\Phi_{i}, \Phi_{j}\right], i \neq j$, connecting two source points of the trajectory. To reconstruct $f(x)$ for some $x \in$ supp $f$ using the chord method, we must first be able to find some chord $C_{i, j}=\left[\Phi_{i}, \Phi_{j}\right]$, such that $x \in C_{i, j}$. For general source trajectories, there can be points $x$ which do not lie on any chord. (Furthermore, even if the trajectory satisfies Tuy's condition for cone-beam reconstruction at the point $x$, even then, a trajectory chord containing $x$ is not ensured [21].)

We assume that $x$ does lie on a suitable chord $C_{i, j}$. Now we let $\alpha_{i, j}=\left(\Phi_{i}-\Phi_{j}\right) /\left\|\Phi_{i}-\Phi_{j}\right\|$ and define $s_{1}, s_{2}$ such that the line segment $A_{i, j}=\left\{x+s \alpha_{i, j} \mid s \in\left[s_{1}, s_{2}\right]\right\}$ is the intersection of the (known) support of $f$ with the chord $C_{i, j}$.

We also assume that $x$ is visible in the detector (not truncated) for the sub-trajectory $\Phi\left(\left[\lambda_{i}, \lambda_{j}\right]\right)$ and we define $h_{1}<0$, $h_{2}>0$ such that $B_{i, j}=\left\{x+h \alpha_{i, j} \mid h \in\left[h_{1}, h_{2}\right]\right\} \subset C_{i, j}$ is the longest line segment containing $x$ which is also visible in the detector for the whole sub-trajectory $\Phi\left(\left[\lambda_{i}, \lambda_{j}\right]\right)$.

For all $y \in B_{i, j}$, it is possible to compute $b_{i, j}(y)$ according to equation (2) with the obvious replacements of 1,2 by $i, j$ and $x$ by $y$. Furthermore, since for all such $y$, there is no truncation along the trajectory segment $\Phi\left(\left[\lambda_{i}, \lambda_{j}\right]\right)$, we are assured that $b_{i, j}(y)=2 H_{\alpha_{i, j}} f(y)$, noting that $y \in C_{i, j}$.

Reconstruction of $f(x)$, or indeed of each $f(y)$ for $y \in$ $B_{i, j}$, depends on the relation between the intervals $\left[s_{1}, s_{2}\right]$ and $\left[h_{1}, h_{2}\right]$. If $\left[h_{1}, h_{2}\right] \subset\left(s_{1}, s_{2}\right)$ then no reconstruction of $f(x)$ is possible; we are in case two as described in section II.B. Otherwise, we are in case one or case three, and reconstruction of $f(x)$ is possible in principle. Moreover, reconstruction of

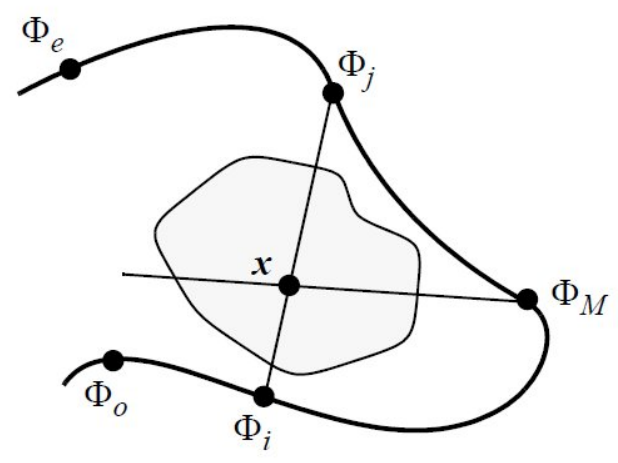

Fig. 1: The source travels along a 3D cone-beam trajectory. The chord $C_{i, j}$ connecting $\Phi_{i}$ to $\Phi_{j}$ is shown, and the M-line from $\Phi_{M}$ passing through $x$ is shown. 
$f(y)$ is possible for any $y=x+s \alpha_{i, j}$ such that $s \in\left[h_{1}, h_{2}\right] \cap$ $\left[s_{1}, s_{2}\right]$ (recalling that the intersection contains at least $s=0$ ).

M-line Reconstruction. If the chord method of reconstruction fails due to case two, insufficient access to $H_{\alpha_{i, j}} f(y)$ for points $y$ on the chord $C_{i, j}$, then reconstruction at $x$ might still be possible by using the M-line approach. A chord passing through $x$ is still needed, but the idea is to use Hilbert inversion along a different line passing through $x$.

We assume as before that $x \in C_{i, j}$ for the chord $C_{i, j}=$ $\left[\Phi_{i}, \Phi_{j}\right]$ and that (a small neighborhood of) $x$ is visible for the sub-trajectory $\Phi\left(\left[\lambda_{i}, \lambda_{j}\right]\right)$. We may now choose any $\lambda_{M} \in$ $\left(\lambda_{i}, \lambda_{j}\right)$ to define the M-line $L_{x, \alpha_{M}}$ where, as before, $\alpha_{M}=$ $\left(x-\Phi_{M}\right) /\left\|x-\Phi_{M}\right\|$. See Fig. 1. In fact, $\lambda_{M}$ can be chosen more generally, provided that $x$ is visible throughout the new sub-trajectory that has been extended by $\lambda_{M}$.

Equation (10) can now be applied to obtain $H_{\alpha_{M}} f(x)$ from the cone-beam projections. As before, we now identify $\left[s_{1}, s_{2}\right]$ such that the line segment $\left\{x+s \alpha_{M} \mid s \in\left[s_{1}, s_{2}\right]\right\}$ is the intersection of the support of $f(x)$ with the M-line $L_{x, \alpha_{M}}$.

In order to obtain $f(x)$ by Hilbert inversion along the M-line, we require $H_{\alpha_{M}} f(y)$ for a range of values of $y$ along this same M-line. Specifically we need $y=x+h \alpha_{M}$ for $h \in\left[h_{1}, h_{2}\right]$ with $h_{1}<0$ and $h_{2}>0$. Each such $H_{\alpha_{M}} f(y)$ can be obtained only if $y$ lies on some chord $C_{k, l}(y)$ which, for a general trajectory, is not necessarily possible in three-dimensions. Furthermore, assuming such a chord $C_{k, l}(y)$ exists, we further require that (a neighborhood of) $y$ be visible in the sub-trajectory containing $\Phi_{k}, \Phi_{l}, \Phi_{M}$.

Once a suitable set of $y$ has been found and $h_{1}$ and $h_{2}$ have been defined, we can subsequently analyze the overlap of the intervals $\left[s_{1}, s_{2}\right]$ and $\left[h_{1}, h_{2}\right]$ to establish whether two-sided inversion, one-sided inversion, or no inversion of the $H_{\alpha_{M}} f$ is possible for the particular M-line chosen.

The advantage of the M-line method is that it provides considerable additional flexibility to choose the direction of Hilbert inversion. The disadvantage is that a range of other points $y$ on the M-line must also lie on their own suitable chords in order to obtain $H_{\alpha_{M}} f(y)$.

The chord method and M-line method both play a role in analyzing helical ROI reconstruction.

\section{HELICAL ROI RECONSTRUCTION THEORY}

\section{A. Chords and $\pi$-lines}

From now on we only consider the helical trajectory which we explicitly write as $\Phi(\lambda)=(R \cos \lambda, R \sin \lambda, p \lambda /(2 \pi))$. Here $p>0$ is the pitch of the helix, and $R>a$ is the radius of the helix cylinder which lies outside the elliptical cylinder $E_{a, b}$ containing the support of $f$. An important and useful property of the helix is that each point inside the helix cylinder lies on a helix chord. This fact can be seen geometrically by considering the following argument. (A different geometric proof was given in [22]).

Take any $x=\left(x_{1}, x_{2}, x_{3}\right)$ with $\|x\|<R$ and let $\Phi\left(\lambda_{1}\right)$ be the intersection of the helix with the "horizontal" plane passing through $x$ (considering the axis of the helix is a vertical line), so $\lambda_{1}=(2 \pi / p) x_{3}$. Now let $\lambda_{0}=\lambda_{1}-\pi$ and $\lambda_{2}=\lambda_{1}+\pi$. Geometrically, it is immediately clear that the line from $\Phi\left(\lambda_{0}\right)$ and passing through $x$ will intersect the helix cylinder at some location $c(0)$ directly above $\Phi\left(\lambda_{1}\right)$ (i.e. $\left.c_{3}(0)>x_{3}\right)$ as shown by a dotted line in Fig. 2. For $t \in[0,1]$ we define $\lambda_{t}=\lambda_{0}+\pi t$ so as $t$ advances from zero to one, $\lambda_{t}$ travels along the helix from $\lambda_{0}$ to $\lambda_{1}$. For each $\lambda_{t}$, the line from $\Phi\left(\lambda_{t}\right)$ which passes through $x$ will strike the inside of the helix cylinder at some location that we define as $c(t)$. When $\lambda_{t}$ reaches $\lambda_{1}$, it is geometrically clear that $c(1)$ is below $\Phi\left(\lambda_{2}\right)$ (in fact, $c_{3}(1)=$ $x_{3}$ ) as shown by another dotted line in Fig. 2. So, as $t$ advanced from $t=0$ to $t=1, c(t)$ traced a continuous curve on the helix cylinder which started above $\Phi\left(\lambda_{1}\right)$ and ended below $\Phi\left(\lambda_{2}\right)$. Therefore, the curve $c(t)$ must intersect the helix at some value of $t^{*} \in(0,1)$ and we identify this intersection point $c\left(t^{*}\right)$ as $\Phi\left(\lambda^{*}\right)$. The helix chord passing through $x$ is $\left[\Phi\left(\lambda_{t^{*}}\right), \Phi\left(\lambda^{*}\right)\right]$.

This construction demonstrated a helix chord through $x$ but there could certainly be other helix chords through $x$ also. Note that $\lambda_{2}>\lambda^{*}>\lambda_{1}>\lambda_{t^{*}}>\lambda_{0}=\lambda_{2}-2 \pi$ so the chord that was constructed satisfies the property that $\lambda^{*}-\lambda_{t^{*}}<2 \pi$, which means that the endpoints of the chord lie on the same "turn" of the helix. Such a helix chord is known as a $\pi$-line [1], and we have demonstrated the well-known fact that all points inside a helix lie on a $\pi$-line. (See [4] for an analytic proof for the existence and uniqueness of $\pi$-lines. We will not make use of the uniqueness property here.)

For source trajectories that are similar to a helix, the same geometric arguments can be applied to demonstrate the existence of $\pi$-lines. For example, if the radius of the helix continuously varied slightly, the same argument could be applied by considering that the modified helix lies on a cylindrical surface that has the same varying radius for the segment $\lambda \in\left(\lambda_{0}, \lambda_{2}\right)$. Other simple variations of the helix can also be handled with the same geometric argument for $\pi$ lines. These small variations in a true helical source trajectory can arise when the scanner uses a flying focal spot (see, e.g., [23]).

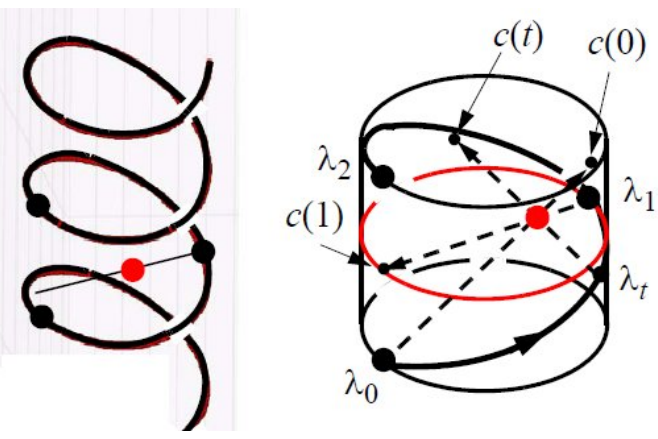

Fig. 2: Demonstration that all points (red dot) inside the helix, lie on a helix cord. Left: The three source points on the helix are spaced by $\pi$ radians. The middle point $\Phi\left(\lambda_{1}\right)$ has the same z-coordinate as the red dot $x$. Right: $\lambda_{t}$ travels from $\lambda_{0}$ to $\lambda_{1}$ and traces a curve from $c(0)$ to $c(1)$ on the inside of the helix cylinder. The point $c(t)$ is found by projecting through point $x$ (red dot) from $\lambda_{t}$ onto the helix cylinder. The curve $c(t)$ must intersect the helix segment from $\lambda_{1}$ to $\lambda_{2}$ thereby identifying the helix chord passing through $x$. 


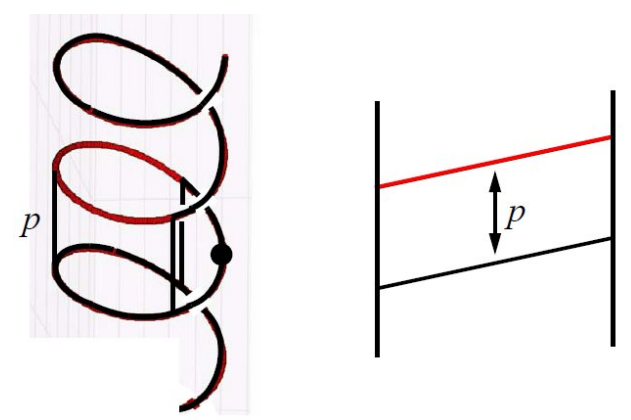

Fig. 3: The Tam-Danielsson window. Left: the "effective" minimal detector, when projected onto the helix cylinder, is bounded by one turn of the helix below (lower detector edge) and one turn of the helix above (upper detector edge) of the corresponding source point. The minimal effective detector height is $P$, the helix pitch. Right: when the cylinder wall is flattened, the effective detector becomes a parallelogram with height $P$. See also Fig. 10, for the Tam-Danielsson window projected onto a planar detector.

\section{B. Full helical reconstruction using the chord method}

Helical image reconstruction generally refers to what was dubbed the "long object problem" [1] where the detector is wide enough to avoid any transverse truncation of the cone-beam projections but the object is longer than the axial extent of the detector. Each point to be reconstructed can be considered to have its own helical sub-trajectory of finite length, corresponding to the trajectory segment for which the point is not truncated (by the axial extent of the detector). In this sense, the long object image reconstruction problem is quite different from the usual situation where all points are exposed to the same source trajectory.

To ensure that all $\pi$-lines remain "visible" (untruncated) in the cone-beam projections, the detector needs to be large enough (axially) to cover the Tam-Danielsson window [1], [2]. The Tam-Danielsson window refers to an effective detector attached to the helix cylinder, which remains fixed with respect to the x-ray source (travels up the helix with the source), and whose height on the cylinder is exactly $p$ (see Fig. 3). If the detector does not satisfy this minimum requirement, then

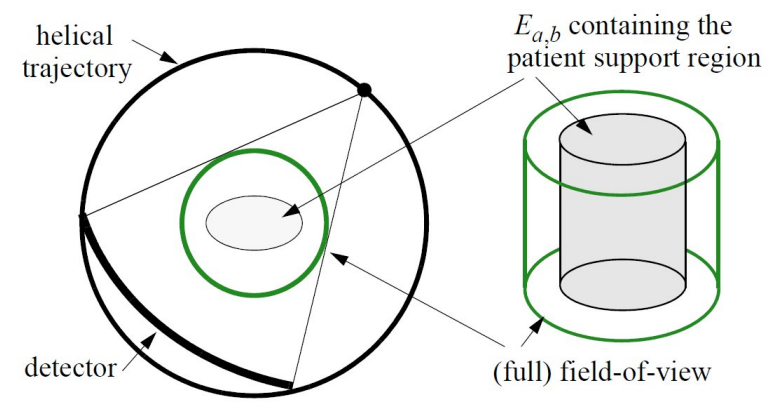

Fig. 4: The full field-of-view (FFOV). Left: top view of the helical trajectory (black circle), the elliptical cylinder $E_{a, b}$, and the cylindrical FFOV (green circle) of radius $r$ determined by the transverse extent of the detector. Right: side view.

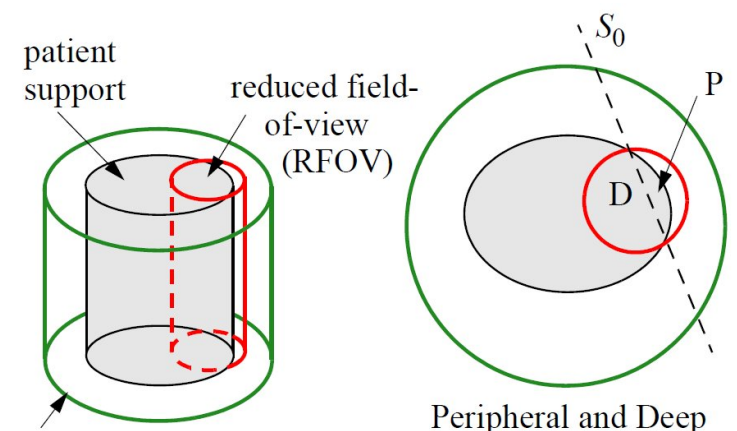

full field-of-view (FFOV)

Fig. 5: The reduced field-of-view (RFOV). Left: the region-ofinterest (ROI) lies inside the RFOV which is the red circular cylinder that intersects the boundary of the patient support region (grey elliptical cylinder). Right: top view. A vertical plane, $S_{0}$ contains the two vertical lines of intersection of the patient support and the RFOV, and this plane cuts the RFOV into the peripheral region $\mathrm{P}$ and the deep region $\mathrm{D}$.

not all $\pi$-lines will be completely measured and Tuy's data sufficiency condition [24] will fail for points whose helical sub-trajectories have no $\pi$-lines.

It is now easy to see that the chord method can be used for helical reconstruction. Each point $x \in E_{a, b}$ lies on a $\pi$-line, which is the chord along which $H_{\alpha} f(x)$ can be computed. Furthermore the expression $b_{1,2}(x)$ can be computed for all $x$ in the cylinder $E_{r, r}$ which is the full field-of-view (FFOV) with $r>a \geq b$, as illustrated in Fig. 4. Therefore $H_{\alpha} f(y)$ is available everywhere along the chord segment $y \in L_{x, \alpha}$ which lies inside the FFOV, and since $E_{a, b} \subset E_{r, r}$, we see that the Hilbert transform is available on bigger line segments that the region of non-zero $f(x)$. We are clearly in "case one" of the two-sided Hilbert transform, which demonstrates that reconstruction is possible in principle, and furthermore, that analytic formulas can be used.

This result on helical reconstruction based on the chord method has been known since 2004 [8].

\section{Helical ROI reconstruction}

The principles behind transverse region-of-interest reconstruction for helical cone-beam tomography are much less obvious. The analysis of this case is the main contribution of this paper.

As illustrated in Fig. 5, the reduced field-of-view (RFOV) inside which the ROI is assumed to lie, is a circular cylinder that intersects the boundary of the support region $E_{a, b}$. (From now on we assume that the ROI fills the RFOV and we use these terms interchangeably.) The cone-beam projections now undergo a dynamic transverse truncation, in such as way that only the lines that pass through the RFOV are measured.

The ROI splits into two regions called "P" for periphery and "D" for deep. They are separated by the vertical plane $S_{0}$ which contains the two parallel vertical lines of intersection of the cylindrical ROI and the support region $E_{a, b}$ as illustrated in Fig. 5 (right). 
Region $P$. It is straight-forward to show that stable image reconstruction is possible inside the $\mathrm{P}$ region. We first use the plane $S_{0}$ to separate the helix into two regions that we illustrate in Fig. 6 as the black and blue components, corresponding to regions $\mathrm{P}$ and $\mathrm{D}$ respectively. We now take any point $x$ inside region $\mathrm{P}$ of the ROI, and consider the two possible kinds of $\pi$ lines that pass through $x$. Since $x$ lies on the same side of $S_{0}$ as the black part of the helix, one endpoint of the $\pi$-line must be a black source point. The two cases, as shown in Fig. 6 are the two possibilities for the other endpoint: either blue (top) or black (bottom).

Now, since $x$ lies in the ROI, it is visible in all projections along its sub-trajectory that connects the endpoints $\Phi_{1}$ and $\Phi_{2}$ of its $\pi$-line, and therefore $H_{\alpha} f(x)=(1 / 2) b_{1,2}(x)$ can be computed from the cone-beam projections along the subtrajectory where $\alpha=\left(\Phi_{2}-\Phi_{1}\right) /\left\|\Phi_{2}-\Phi_{1}\right\|$ is the direction of the $\pi$-line. Similarly, for any $y=x+s \alpha$ lying on the same $\pi$-line with $y$ inside the RFOV, $H_{\alpha} f(y)$ can be obtained from the projections.

To examine the possibilities for finite Hilbert inversion, we need the intervals $\left[s_{1}, s_{2}\right]$ and $\left[h_{1}, h_{2}\right]$ and these are readily seen from the figure. The interval $\left[s_{1}, s_{2}\right]$ is the intersection of the grey elliptical region $E_{a, b}$ with the $\pi$-line $L_{x, \alpha}$ and the
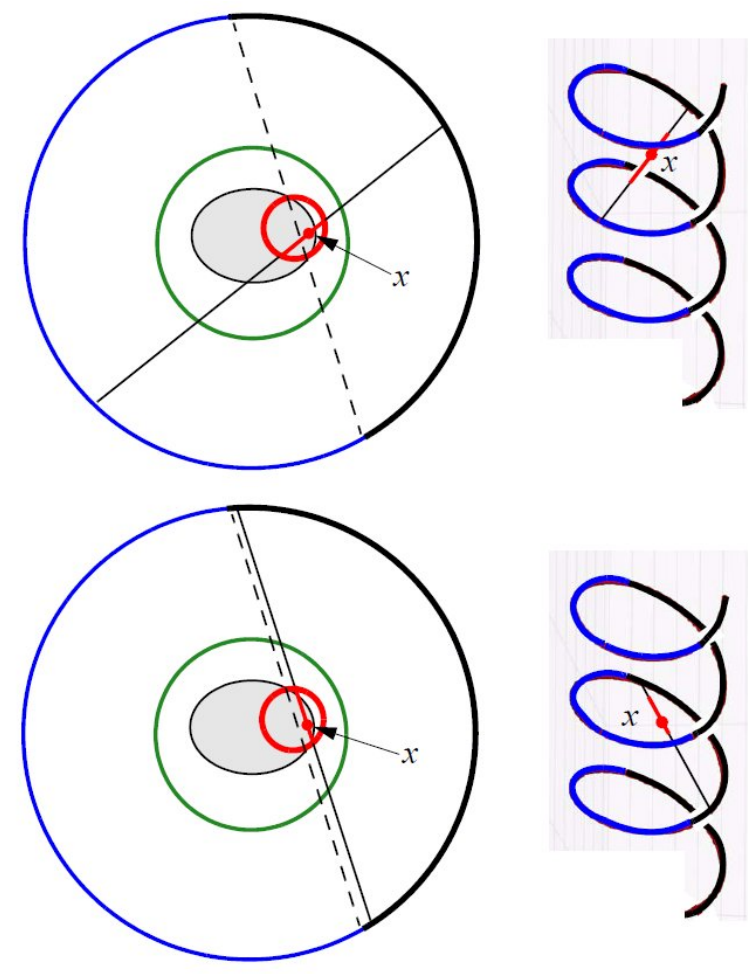

Fig. 6: ROI reconstruction in region P. Top: (left) a point $x$ is taken in region $\mathrm{P}$, whose $\pi$-line (right) connects a black helix point to a blue helix point. Reconstruction of $f(x)$ can be achieved using one-sided finite Hilbert inversion. Bottom: (left) a point $x$ in region $\mathrm{P}$ has a $\pi$-line (right) connecting two black points of the helix. The two-sided finite Hilbert inversion formula can be used to reconstruct $f(x)$. (The righthand figures are strictly schematic, and not in scale with the left-hand counterparts.) interval $\left[h_{1}, h_{2}\right]$ is the intersection of the cylindrical RFOV with this same line. We immediately note that if both endpoints of the $\pi$-line are black helix points, then two-sided Hilbert inversion is possible, and if only one endpoint is black, then the one-sided inversion can be used. Thus every point $x$ inside region $\mathrm{P}$ can be reconstructed in principle.

Region $D$. The approach for region $\mathrm{D}$ is a little different. For each point $x$ in region $\mathrm{D}$, we define two vertical planes $S_{1}(x)$ and $S_{2}(x)$, where $S_{1}$ contains $x$ and passes through one (vertical) line of intersection between the RFOV and $E_{a, b}$, and $S_{2}$ is defined similarly for the other line of intersection. See Fig. 7. (To simplify the notation, we drop the $x$ dependence for $S_{1}$ and $S_{2}$.) The two planes are then used to subdivide the helix into alternating segments which we conceptually label as black or blue, where the black segments are exactly those helix points that are intersected by vertical planes passing through $x$ and the part of the boundary of $E_{a, b}$ that remains inside the

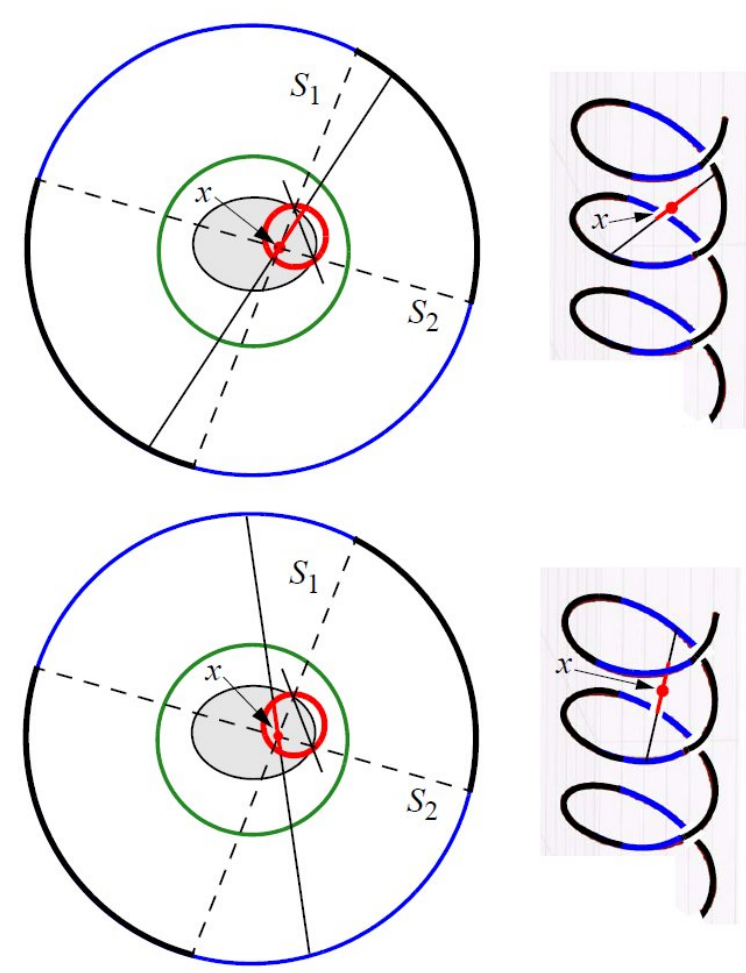

Fig. 7: Chord reconstruction in region D. Top: (left) fix an arbitrary location $x$ inside region D. The planes $S_{1}$ and $S_{2}$ are defined so that a line between them and passing through $x$ will exit the support of $f$ before exiting the (red, circular) RFOV. The black part of the helix is labeled accordingly. The $\pi$-line through $x$ (right) connects two black helix points, so the Hilbert transform can be obtained for all (red) points along the $\pi$-line that are also inside the RFOV. Reconstruction of $f(x)$ can be achieved using one-sided finite Hilbert inversion. Bottom: (left) a point $x$ in region $\mathrm{D}$ has a $\pi$-line (right) connecting two blue points of the helix. For this point $x$, the chord method fails for reconstruction of $f(x)$, because the (red) chord segment inside the RFOV is completely contained inside the (grey) support of $f$. 

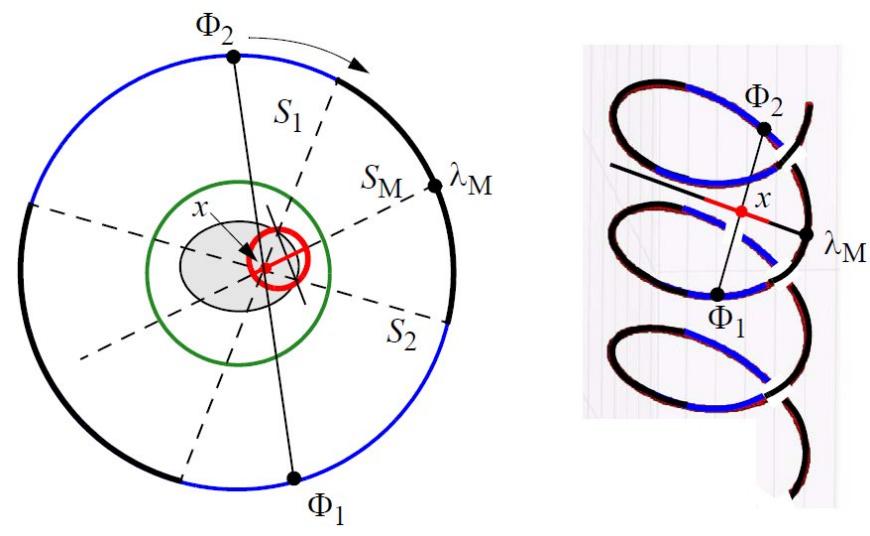

Fig. 8: Finding an appropriate M-line. First the plane $S_{M}(x)$ is defined to lie between $S_{1}$ and $S_{2}$. Then, descending the helix from $\Phi_{2}$ towards $\Phi_{1}$, the intersection of the helix with $S_{M}$ is selected for $\lambda_{M}$. Note that $\Phi_{M}$ must lie on a black helix segment by this construction.

\section{RFOV}

This situation now splits into an "easy" case and a "hard" case. We examine the $\pi$-line passing through the point $x$. By construction, this $\pi$-line will either have two blue endpoints or two black endpoints. We label these endpoints as $\Phi_{1}$ and $\Phi_{2}$. If the two endpoints are black (easy case) then, following the same reasoning as was given for region $\mathrm{P}$, the $\pi$-line is suitable for reconstruction of $f(x)$ using the chord method. For all points $y$ along the intersection of the $\pi$-line and the RFOV (the red segment in Fig. 7 (top)), the Hilbert transform $H_{\alpha} f(y)$ can be obtained from the differentiated backprojection along the trajectory from $\Phi_{1}$ to $\Phi_{2}$. (Here $\alpha$ is the direction of the $\pi$-line.) The one-sided inverse Hilbert transform can then be applied along this chord segment to obtain the reconstructed value $f(x)$ (and incidentally any $f(y)$ along the chord segment).

If the two endpoints are blue (hard case), then the chord method cannot be applied because the Hilbert transform $H_{\alpha} f(x)$ can only be computed inside the RFOV which remains interior to the support region $E_{a, b}$. Visually, in Fig. 7 (bottom), the red segment of the chord (with blue endpoints) is completely contained inside the object (shaded grey). We are therefore in case 3 for Hilbert inversion (see section II.B.) where $\left[h_{1}, h_{2}\right] \subset\left(s_{1}, s_{2}\right)$ which corresponds to loss of uniqueness of the finite Hilbert transform. This particular point $x$ cannot be reconstructed using the chord method.

For this situation of two blue endpoints (say, $\Phi_{1}$ and $\Phi_{2}$ ) for the $\pi$-line through $x$ inside region $\mathrm{P}$, we need to invoke the M-line method to establish that reliable reconstruction of $f(x)$ can be obtained. The idea is to choose a suitable helix point $\lambda_{M}$ such that the corresponding M-line through $x$ will exit the support of $f$ before exiting the RFOV (i.e. that the M-line behaves the same way as the $\pi$-line in Fig. 7 top). Furthermore we must ensure that $H_{\alpha_{M}} f(y)$ can be computed for all $y$ on the intersection of this M-line with the RFOV, taking into account the potential axial truncation due to the Tam-Danielsson detector window.
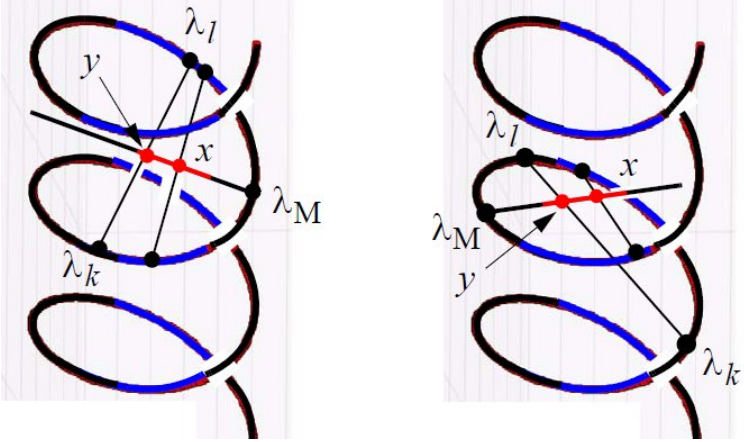

Fig. 9: Possible $\pi$-lines through points $y$ on the chosen Mline through $x$. Left: the $\pi$-line $C_{k, l}(y)=\left[\Phi_{k}, \Phi_{l}\right]$ is located so that $\lambda_{M} \in\left(\lambda_{k}, \lambda_{l}\right)$. Right: for a different $x$ and different M-line, a point $y$ might conceivably have its $\pi$-line located so that $\lambda_{M} \notin\left(\lambda_{k}, \lambda_{l}\right)$. This case turns out to not be geometrically possible. See text.

To this end, we first define an M-plane $S_{M}(x)$ passing through $x$ and bisecting the angle between two planes $S_{1}(x)$ and $S_{2}(x)$ as illustrated in Fig. 8. The point $\Phi_{M}$ is chosen as follows. Starting from the higher $\pi$-line endpoint $\Phi_{2}$, descend the helix towards $\Phi_{1}$. Before reaching $\Phi_{1}$, one must pass through the intersection of the helix with the plane $S_{M}$, and this intersection point is chosen as $\Phi_{M}$, as shown in Fig. 8. By this construction, we have $\lambda_{M} \in\left(\lambda_{1}, \lambda_{2}\right)$. Defining $\alpha_{M}=\left(x-\Phi_{M}\right) /\left\|x-\Phi_{M}\right\|$ as usual, we can now compute $H_{\alpha_{M}} f(x)$ from $\Phi\left(\left[\lambda_{1}, \lambda_{2}\right]\right)$ using formula (10) bearing in mind that $x$ is visible (not truncated transversely or axially) for all projections along $\left[\lambda_{1}, \lambda_{2}\right]$.

We must now show that $H_{\alpha_{M}} f(y)$ can be computed for all $y$ along the intersection of this M-line with the RFOV. Take any such $y$, and let its $\pi$-line be denoted $C_{k, l}(y)=\left[\Phi_{k}, \Phi_{l}\right]$ (where $\left.\lambda_{k}<\lambda_{l}\right)$. Now if $\lambda_{M} \in\left(\lambda_{k}, \lambda_{l}\right)$ (as shown in Fig. 9 (left)), then $H_{\alpha_{M}} f(y)$ can be computed using $\Phi\left(\left[\lambda_{k}, \lambda_{l}\right]\right)$ because (a neighborhood of) $y$ is visible in all the projections $\lambda \in\left(\lambda_{k}, \lambda_{l}\right)$ (within the axial truncation defined by the Tam-Danielsson window, and within the transaxial truncation of the RFOV). It turns out that the case shown in Fig. 9 (right), where $\lambda_{M} \notin$ $\left(\lambda_{k}, \lambda_{l}\right)$, cannot occur, as we show below. The demonstration that helical ROI reconstruction is possible is complete when this detail has been established.

One way to demonstrate that $\lambda_{M} \in\left(\lambda_{k}, \lambda_{l}\right)$ for any $y$ with corresponding $\pi$-line $C_{k, l}(y)$ is to consider the CB projection at the source point $\Phi_{M}$. The essential fact is that the points $x$ and $y$ project to the same detector location (because they lie on a common M-line from $\Phi_{M}$ ). For the argument we use here, it is more convenient to consider a planar detector, which we conceptually place tangentially to the helix cylinder. The upper and lower boundaries of this detector are the CB projections of one turn of the helix respectively above and below the source point $\Phi_{M}$ (accounting exactly for the Tam-Danielsson detector window but ignoring the RFOV transverse truncation). We now consider the $\mathrm{CB}$ projection of the $\pi$-line $C_{1,2}$ through $x$, noting that since $\lambda_{M} \in\left(\lambda_{1}, \lambda_{2}\right)$, this projection will be a line segment with one endpoint at the top of the detector, 


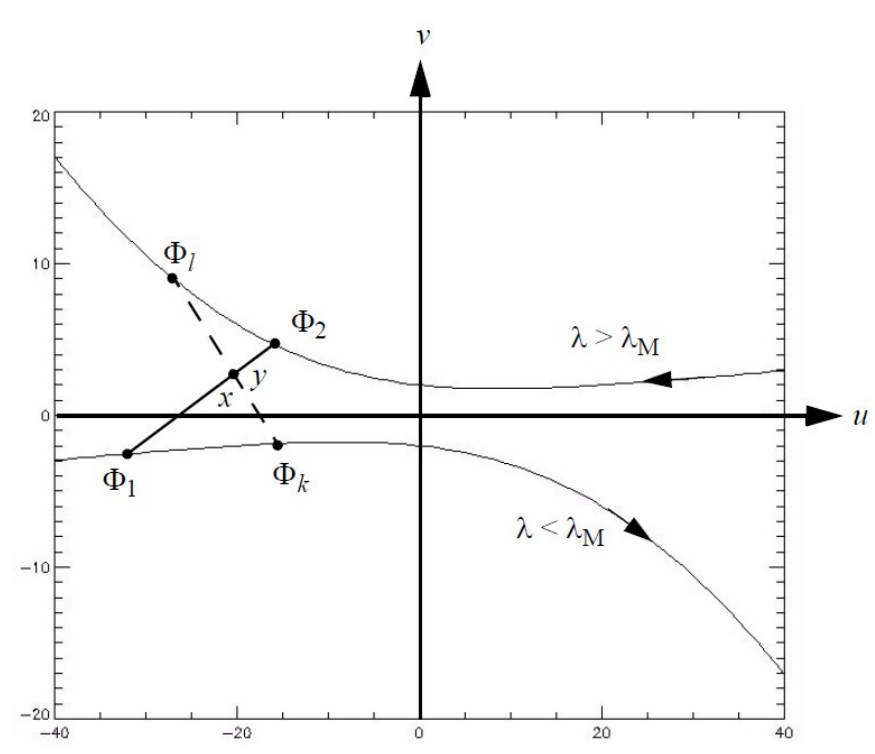

Fig. 10: The flat detector plane of the cone-beam projection $\Phi_{M}$. The space between the two curves is the Tam-Danielsson window, with the upper curve being the projection of the helix turn above $\Phi_{M}$, and the lower curve from the helix turn below $\Phi_{M}$. Since $\lambda_{M} \in\left(\lambda_{1}, \lambda_{2}\right)$, we know the projections of $\Phi_{1}$ and $\Phi_{2}$ lie on the lower and upper curves respectively. Since $y$ projects to the same point as $x$, its $\pi$-line $C_{k, l}(y)$ must also have one endpoint on each curve, such as the dashed line suggested in the figure. The curves are drawn to scale, using $R=10, p=4$, and detector extent $80 \times 40$.

and one endpoint at the bottom; the projection of the point $x$ lies inside the projection $g\left(\lambda_{M}, \cdot\right)$. Now, the projection of the $\pi$-line $C_{k, l}(y)$ must intersect the projection of the point $x$ on the detector (since $x$ and $y$ project to the same detector location). The endpoints of $C_{k, l}(y)$ are on the upper or lower detector curves, and the only possibility is that there must be one endpoint on each. See Fig. 10. The upper curve is the projection of the helix turn that lies above $\Phi_{M}$ and the lower curve is the projection of the helix turn lying below $\Phi_{M}$ and therefore $\lambda_{M} \in\left(\lambda_{k}, \lambda_{l}\right)$ as required.

The concave up and concave down shape of these two detector curves are vital to the argument. The mathematical form of the upper ("top") curve $v_{T}$ is given by

$$
\begin{aligned}
v_{T}(u) & =\frac{u^{2}+(2 R)^{2}}{(2 R)^{2}} \frac{\lambda}{2 \pi} p \\
\text { where } \quad \frac{\lambda}{2} & =\arccos \frac{u}{\sqrt{u^{2}+(2 R)^{2}}}
\end{aligned}
$$

(in agreement with [2] and [4]). The lower ("bottom") curve is given by $v_{B}(u)=-v_{T}(-u)$. From these two expressions it can be verified that $v_{T}^{\prime \prime}(u)>0$ and $v_{B}^{\prime \prime}(u)<0$, which proves the concave behavior of the curves.

In this subsection, we have shown that ROI reconstruction within a RFOV from helical projections is possible in principle, using one-sided or two-sided finite Hilbert inversion. The RFOV was split into regions $\mathrm{P}$ and $\mathrm{D}$. All points inside region $\mathrm{P}$ can be reconstructed using standard $\pi$-line reconstruction with one-sided Hilbert inverse, or in a few cases, using the
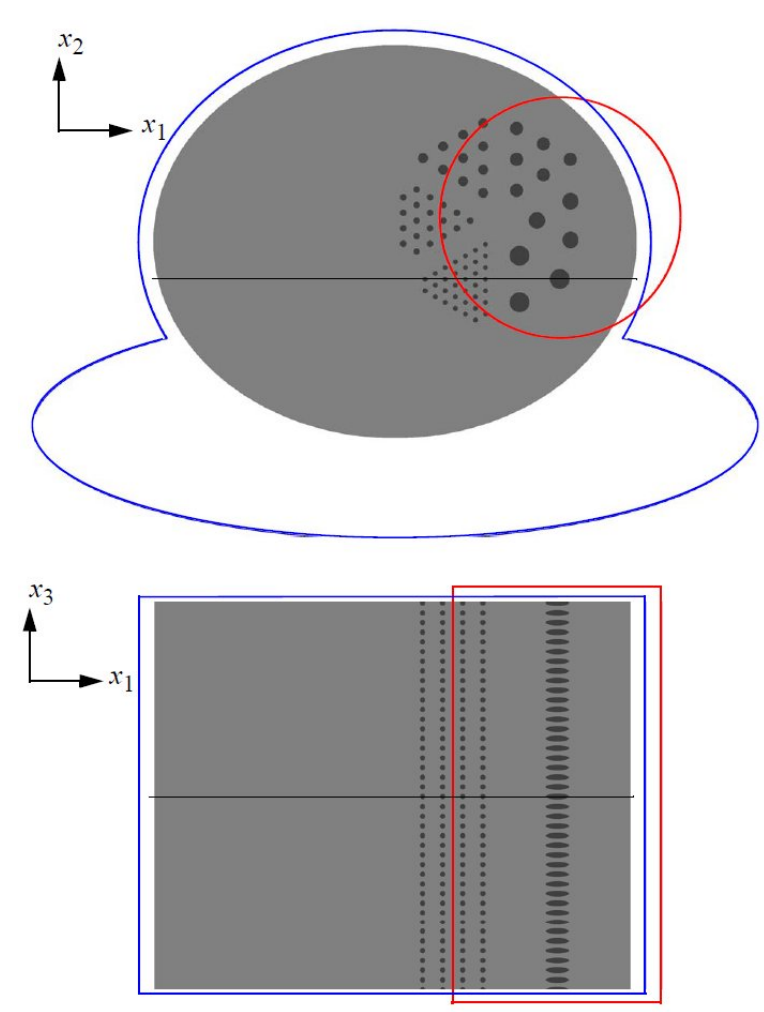

Fig. 11: Illustration of the simulation phantom with the RFOV (red) and the support regions (blue) shown. The phantom contrast was $1 \%$. Top: axial slice $x_{3}=128$. Bottom: coronal slice $x_{2}=171$. The intersection of the two slices is indicated with the horizontal line in each slice. All images of computer phantoms are displayed with compressed greyscale range $[0.98,1.02]$.

two-sided Hilbert inversion, determined by the orientation of the $\pi$-line passing through the point in question. For region $\mathrm{D}$, some points can be reconstructed according to the one-sided Hilbert inverse if their $\pi$-lines are in a particular geometric configuration. For the other points, the standard chord method ( $\pi$-line method) cannot be used because the $\pi$-line results in a geometry of the "interior" finite Hilbert transform (case 2 in section IIB). However, for this last group of points, it is possible to apply the M-line approach, to allow one-sided finite Hilbert inversion.

In the next section, we provide example reconstructed images from ROI helical scans.

\section{EXAMPLES OF HELICAL ROI RECONSTRUCTION}

To illustrate ROI reconstruction from a helical trajectory, we performed three reconstructions. Two reconstructions were from simulated data, and one was from real patient data. The same projection geometry, reconstruction parameters, and reconstruction algorithm were used for all three reconstructions.

The projection data corresponded to 3,091 source positions taken along $4 \times 360^{\circ}+8.4^{\circ}$ (just over 4 helix turns) at a radius that varied between $595 \mathrm{~mm}$ and $600 \mathrm{~mm}$ due to the flying focal spot. The helix pitch was $p=23 \mathrm{~mm}$. The cylindrical detector of $245 \times 32$ pixels had a transverse extent of $945 \mathrm{~mm}$ 


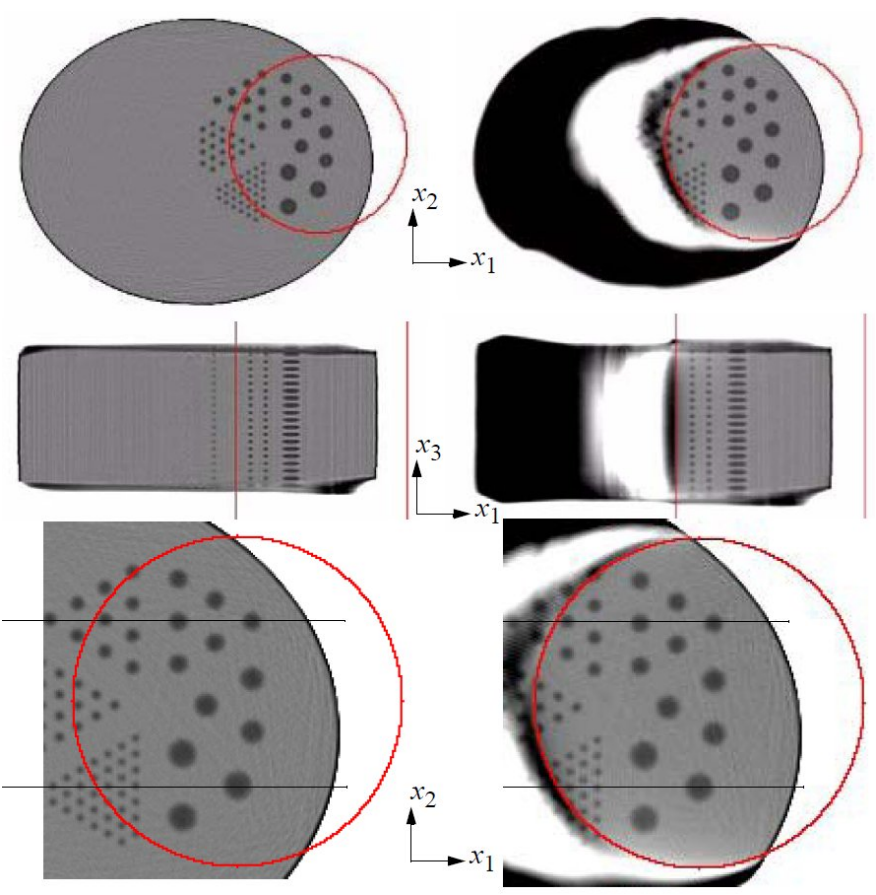

Fig. 12: Full-field and RFOV reconstructions from noisefree simulated data. Left column: full-field projection data, with no transverse truncation: axial and coronal slices of the reconstructed phantom, and a zoom of the ROI. Right column: reduced field-of-view (RFOV) projection data with transverse truncation. The red lines indicate RFOV boundary, no measured lines were obtained unless they intersected the red cylinder. In the zoomed images, the two horizontal lines are at $x_{2}=250$ (upper line) and $x_{2}=170$ (lower line) and they indicate the level of the line plots shown in figure 13 .

and axial extent of $70 \mathrm{~mm}$. The resulting cylindrical FFOV had a diameter of $501 \mathrm{~mm}$. The RFOV was defined as a cylinder of diameter $160 \mathrm{~mm}$ with central axis passing through $\left(x_{1}, x_{2}\right)=$ $(112,38)$. All coordinates are given in units of millimeters. The green FFOV and red RFOV shown in the left of Figs. 6, 7, 8 are drawn true to scale with respect to the helical trajectory.

The reconstruction procedure was not based on the DBP approach described in this paper. The theory section established that helical ROI data is sufficient for stable image reconstruction, so in principle, any algorithm that correctly models the system should be successful. All the reconstructions presented here were carried out using the conjugate gradient algorithm implemented in the Reconstruction Took Kit (RTK) [25] to perform least-squares minimization of the data term with a light regularization term consisting of the squared norm of the gradient of the object function. For simplicity, the same regularization parameter was used for all reconstructions even though the FFOV data involved about twice as many data points (twice as many line-integrals) passing through the object as the RFOV case, and even though the noise levels in the projections varied considerably from noise-free simulations to real patient data. The reconstructions were obtained on a regular grid of $1 \mathrm{~mm} \times 1 \mathrm{~mm} \times 1 \mathrm{~mm}$ voxels of dimensions 478 x 338 x 256 (256 axial slices). An approximate support region
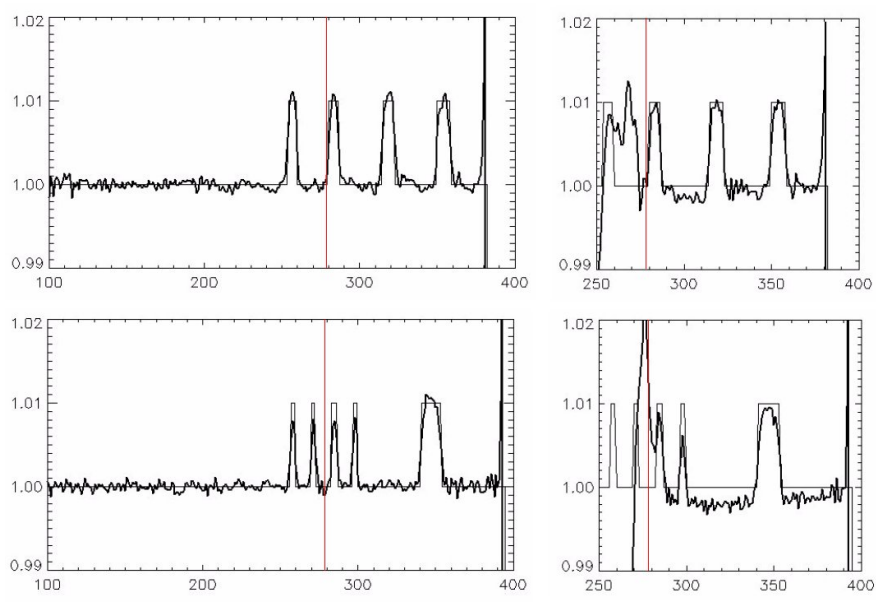

Fig. 13: Line profiles through the reconstructed images of the noise-free mathematical phantom. Axial slice $x_{3}=128$. Top: profiles through the line $x_{2}=250$ (see Fig. 12). Bottom: profiles through the line $x_{2}=170$. Left column: image reconstructed from the FFOV (see Fig. 12(left)). Right column: image reconstructed from projection data truncated to the RFOV (see Fig. 12(right)). The red vertical lines indicate the boundary with the RFOV; in principle, the reconstructions should be identical on the right of the red line.

was defined, outside which a density of zero was assumed. The support region consisted of the union of two elliptical cylinders $E_{170,140}$ centered at $\left(x_{1}, x_{2}\right)=(3,22)$, and $E_{240,75}$ centered at $(3,-100)$. The second elliptical cylinder accounts for the patient bed. For all reconstructions, one thousand iterations were run, having verified with preliminary studies that only very small improvements (barely discernible) were observed when running 3,000 iterations.

\section{A. Computer simulated data}

For the two simulation studies, the mathematical phantom consisted of an elliptical cylinder centered transaxially at $(3,22)$, and with transaxial half axis lengths $160 \mathrm{~mm}$ and $130 \mathrm{~mm}$ in the $x_{1}$ and $x_{2}$ directions respectively. The elliptical cylinder had uniform density 1.0 units. Inside this cylinder, a pie resolution phantom was superimposed, with incremental density 0.01 units (one percent contrast). The pie phantom had six segments, each containing a collection of identical uniform circles. The largest circles were of diameter $15.36 \mathrm{~mm}$ and there were 3 such circles in the segment. The smallest circles were of diameter $3.84 \mathrm{~mm}$, with 34 such circles in that segment. This pie phantom was positioned so that the smaller resolution features would lie at the inner boundary of the RFOV and the patient support region. In the axial direction, the resolution features were elliptical with the same axis length of $3.84 \mathrm{~mm}$. This pie phantom was repeated at different $x_{3}$ levels, spaced axially every $6.4 \mathrm{~mm}$, so the gap between successive phantoms was $2.56 \mathrm{~mm}$.

Fig. 11 illustrates the mathematical phantom, and the position of the pie resolution phantom with respect to the RFOV. Also shown is the support region, the union of two support cylinders of elliptical cross-section. 
The projections of the mathematical phantom were simulated using analytic line-length calculations through the component ellipsoids and multiplying by the uniform density. Nine ray-sums were averaged for each detector pixel. For the RFOV projections, only those rays that intersected the cylindrical RFOV were calculated.

The reconstructed images for the first phantom study are shown in Fig. 12. Visually, the ROI was reconstructed with them same quality as the full-field case, with all the same resolution units visible. Outside the RFOV cylinder, the reconstructed image quality immediately degraded. The line profile plots of Fig. 13 provide more quantitative information and show that the ROI reconstructions are about $0.2 \%$ less accurate quantitatively than the FFOV reconstruction. More discussion of this point is provided in section $\mathrm{V}$ below.

The second phantom data case used the same simulated projections as the first case, but with Poisson noise added. The noise level added corresponded to $10^{7}$ photons being transmitted for each detector pixel. The phantom densities were scaled by $0.01879 / \mathrm{mm}$ and the maximum level of attenuation resulted in roughly $24 \times 10^{3}$ detected photons. The Poisson noise was included after applying the Beer Lambert law to the line integrals, and before returning to line-integral values using the standard logarithmic processing. Note that the maximum level of Poisson noise in the measurement data was well under $1 \%$ (before logarithms).

In Fig. 14, the same zoomed axial slices of the reconstructed images are shown as for the noise-free case, and the same lines were used as in Fig. 13 for the profile plots. Note that the same noise realization was used for both the FFOV and RFOV projection data, which explains why the noise features inside the ROI of the two reconstructed images were so similar. We also observe that at this noise level, the small difference between FFOV and RFOV reconstructions was completely dominated by the fluctuations in the reconstructed images.

\section{B. Real patient data}

The real patient data were taken from training case L067 data supplied for the "Low Dose CT Grand Challenge, 2015" [26]. For our purposes, we extracted a subset of the full patient data supplied. The 3,091 source positions were subsampled along roughly 4 turns of the 20 turns of helix data available, and the detector pixel values $(245 \times 32$ pixels $)$ were also subsampled from the original clinical scan data.

Similar to the simulated noisy data example, the RFOV projections were obtained by taking the full (FFOV) projection patient data and removing all rays that did not intersect the cylindrical RFOV. Consequently, the RFOV data was strictly a subset of the FFOV data, and included exactly the same statistical and systematic noise.

A transverse and a coronal slice through the reconstructed FFOV and RFOV images from patient data are shown in Fig. 15. We observe the same behavior as for the simulated phantom data: the image inside the ROI region of both reconstructions appears to be visually identical, whereas outside the ROI the reconstruction from RFOV data degrades almost immediately, with none of the features of the FFOV reconstruction visible a short distance outside the ROI. However,
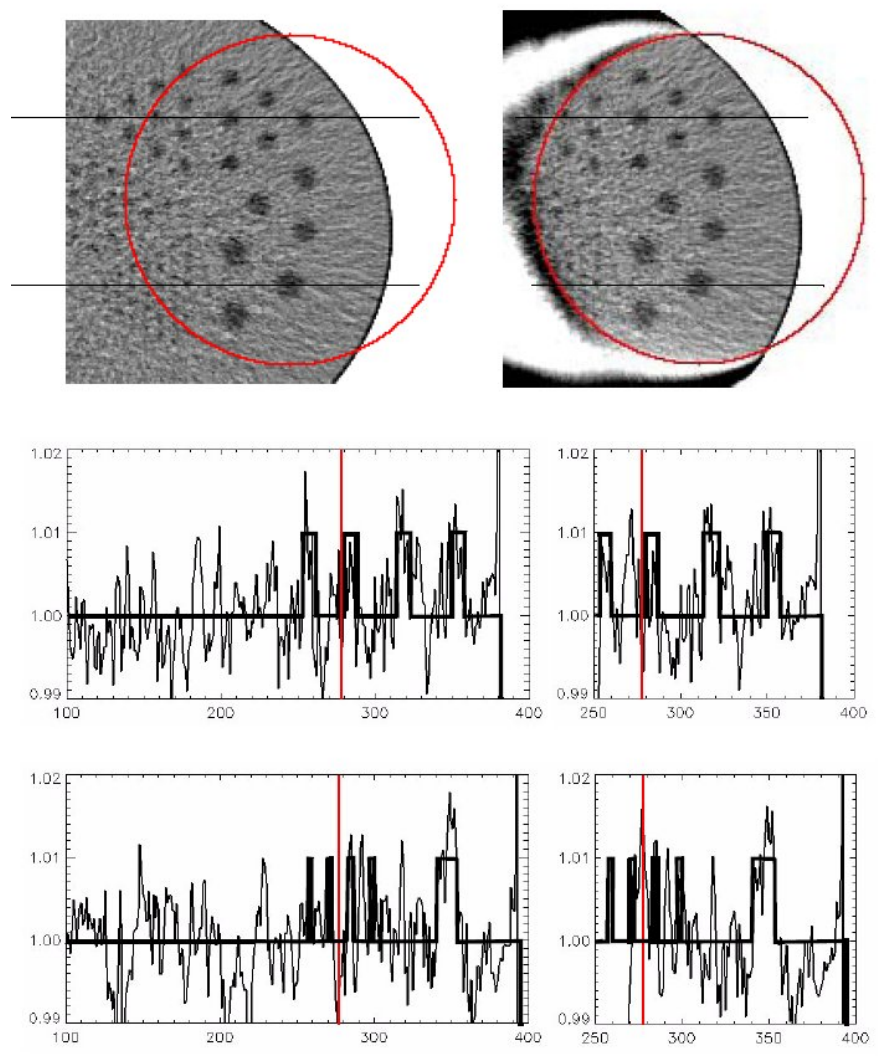

Fig. 14: Reconstructed images from noisy synthetic projection data. Left column: full field-of-view (FFOV). Right column: reduced field-of-view (RFOV). Top row: greyscale images of axial slice $x_{3}=128$. Middle and bottom rows: profile plots along the lines $x_{2}=250$ and $x_{2}=170$ respectively. The red vertical lines indicate the boundary with the RFOV.

line profiles shown in Fig. 16 indicate that the images inside the ROI are not identical. The density amplitudes of the two reconstructions diverge from each other while still inside the cylindrical ROI. For the two profiles shown, this diverging behavior occurs only near the edge of the ROI at the side where the RFOV is "interior" (not near the boundary of the patient cross-section). We observed similar behavior for profiles taken at other locations (not shown here).

\section{Discussion AND CONCLUSIONS}

We have used existing image reconstruction theory to establish that transverse truncation of projections to form a RFOV still allows mathematically correct image reconstruction for ROIs inside the RFOV. Accurate image reconstruction for conventional (FFOV) helical scanning with axial truncation can be demonstrated using the chord method and the two-sided finite inverse Hilbert transform. The two main components of our mathematical demonstration for helical RFOV scanning are (i) that the use of the one-sided inverse Hilbert transform is essential and (ii) that the M-line method needs to be invoked because chords alone would not satisfy the geometric requirements for the inverse Hilbert transform theory. The conclusion is that for a convex RFOV that intersects the 


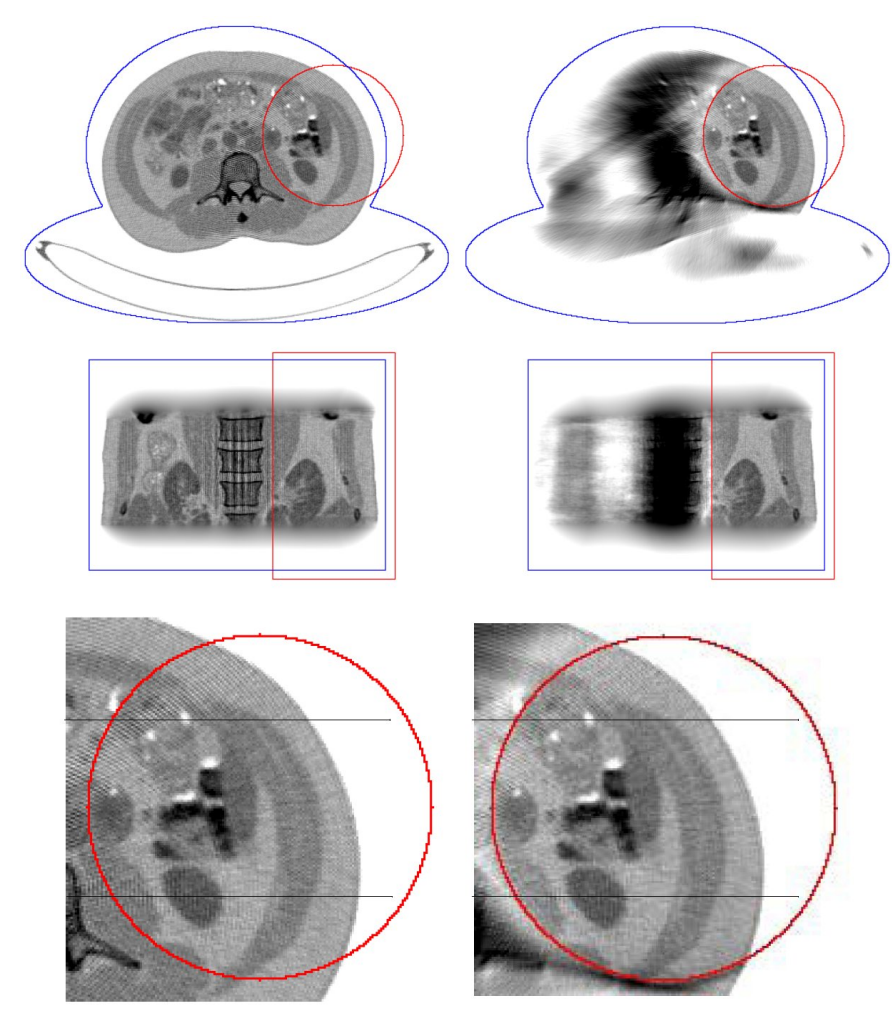

Fig. 15: Reconstructed images from real patient data. Left column: images from FFOV projections; right column: images from the RFOV projections. Top row: slice $x_{3}=128$ of the reconstructed images, displayed with the assumed object support indicated in blue, and the boundary of the RFOV in red. Middle row: slice $x_{2}=171$ of the reconstructed images, with the support region and RFOV boundary shown. Last row: zoomed axial images of slice $x_{3}=128$.

(assumed known) patient boundary, helical projection data that is truncated axially according to the Tam-Danielsson window, and transversely according to the RFOV is sufficient for accurate image reconstruction.

No analytic reconstruction algorithm was proposed here. A major difficulty in constructing such an algorithm is the inversion of the one-sided finite Hilbert transform. This inversion is known to be unique and stable, but no explicit formula or direct algorithm currently exists to perform the inversion. The singular value decomposition (SVD) of the one-sided finite Hilbert transform has been under intense investigation recently, with new results on the spectrum of this operator and the general behavior of the singular functions [17]-[19]. However, an explicit analytic expression of this SVD remains elusive, as do other analytic descriptions of the inverse.

A reconstruction algorithm could be formulated that reduces the three-dimensional image reconstruction problem to a large set of one-dimensional Hilbert inversions, following, for example, the ideas in Schoendube et al [27] (who used two-sided Hilbert inversions since transverse truncation was not considered there), with the computational and storage advantage that a large three-dimensional set of voxels enclosing the full cross-sectional support of the object would not be necessary.
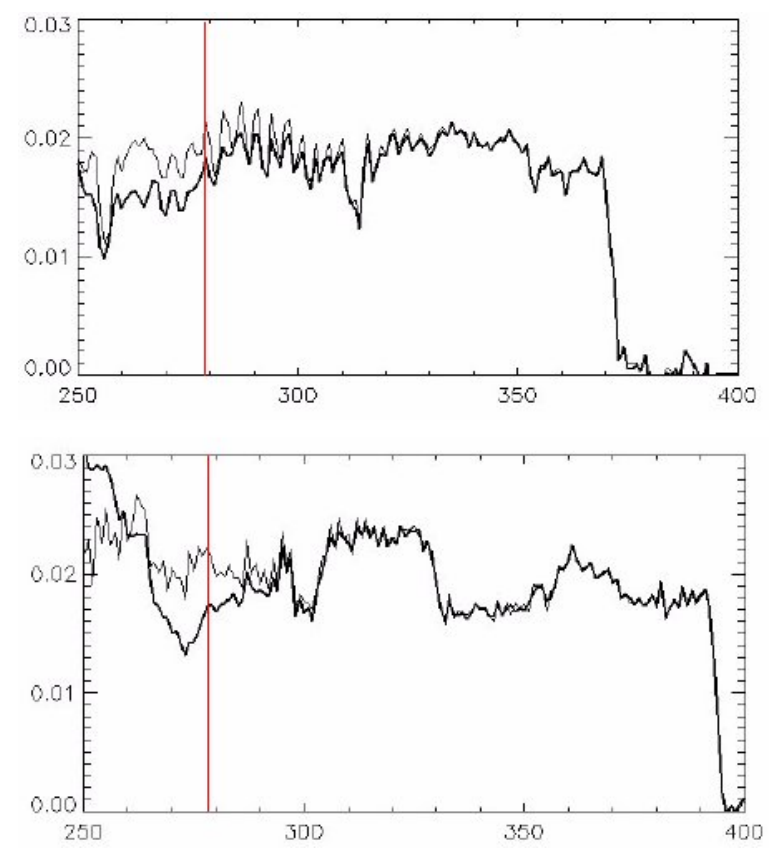

Fig. 16: Two line profiles through the reconstructed images from real patient data. The position of the lines corresponding to the two plots are shown in Fig. 15. The thin lines show the reconstructed values from the FFOV, and the thick lines correspond to the RFOV reconstructions. The red vertical lines indicate the boundary with the RFOV.

The resulting one-dimensional Hilbert inversions would be of moderate size, with matrix dimensions of (at most) a few thousand on each side. Direct digital inversion using SVD or other methods would therefore be feasible. Alternatively, the finite Hilbert inversions could be performed using a fast iterative method for solving linear equations.

We presented reconstructed images from noise-free and noisy phantom data, and from real patient data. When compared to the reference reconstructions created using full-field (FFOV) projection data, we found that the ROI from the reduced-field (RFOV) projections was visually identical to the same region in the reference images. However, when examining the intensity levels using the profile plots, we found that the RFOV reconstructions inside the ROI were not as accurate as the FFOV intensities. The general tendency was that the images were identical for ROI regions near the patient/phantom boundary, but the amplitudes diverged near the interior boundary of the ROI (see Figs. 13 and 16). We conjecture that this behavior is related to the relative inherent stability of the two image reconstruction problems. The FFOV reconstruction problem can be resolved using the stable two-sided Hilbert inversion, whereas the RFOV required the less stable one-sided finite Hilbert inversion (at least, in our mathematical demonstration). Defrise et al [15] established that inversion of the one-sided finite Hilbert transform is stable, but that the stability constant degrades rapidly near the "inner" boundary (see section IIB). These inner boundaries of the one-dimensional Hilbert transforms would occur at the interior part of the ROI boundary, and this region is where 
we observed the greatest discrepancy between the RFOV and FFOV reconstructions. A poor stability constant means that the reconstruction would be more susceptible to errors or inconsistencies in the projection measurements. The real patient data has more complicated noise patterns due to a number of physical effects, so it is reasonable to expect that the real data would produce more marked effects than the two simulations studies. This same argument was put forth in [28] as a possible explanation for similar behavior observed in their ROI reconstructions.

We emphasize here that although much is known about the one-sided finite Hilbert transform operator, we do not have direct information on the properties of the helical ROI problem. We used the finite Hilbert transform to show that helical ROI reconstruction is possible in principle, but there may be other (yet unknown) ways to demonstrate this result. The stability properties of the inverse finite Hilbert transform provide a worst case scenario, and seem to be in general agreement with our observations, but the fundamental reasons for the discrepancies observed in Figs. 13 and 16 may turn out to be much more subtle.

In summary, we have demonstrated that transverse ROI imaging with helical trajectories admits mathematically accurate image reconstruction. The small intensity differences when compared to the reference scan is compatible with known theory for inversion of the one-sided Hilbert transform. Until these effects are more clearly understood, care must be taken with ROI reconstruction results for regions where the ROI lies close to the interior wall of the RFOV.

\section{APPENDIX}

In this appendix we demonstrate equation (7). This equation is fundamental for the chord and M-line methods which were essential to Section III. Equation (7) has been presented in a slightly different form in [12] and a 2D version was presented in [9]. An earlier demonstration using a different approach was given in [8] but it only demonstrated the chord form, equation (8).

Here we write $\Phi(\lambda)$ as $\Phi_{\lambda}$, for short. Starting from equations (1) and (2) we have

$$
\begin{aligned}
& b_{1,2}(x) \\
& =\left.\frac{1}{\pi} \int_{\lambda_{1}}^{\lambda_{2}} \frac{1}{\left\|x-\Phi_{\lambda}\right\|} \frac{\partial}{\partial \lambda} \int_{0}^{\infty} f\left(\Phi_{\lambda}+t \theta\right) d t\right|_{\theta=\frac{x-\Phi_{\lambda}}{\left\|x-\Phi_{\lambda}\right\|}} d \lambda \\
& =\left.\frac{1}{\pi} \int_{\lambda_{1}}^{\lambda_{2}} \frac{1}{\left\|x-\Phi_{\lambda}\right\|} \int_{-\infty}^{\infty} \Phi_{\lambda}^{\prime} \cdot \nabla f\left(\Phi_{\lambda}+t \theta\right) d t\right|_{\theta=\frac{x-\Phi_{\lambda}}{\left\|x-\Phi_{\lambda}\right\|}} d \lambda \\
& =\frac{1}{\pi} \int_{\lambda_{1}}^{\lambda_{2}} \int_{-\infty}^{\infty} \Phi_{\lambda}^{\prime} \cdot \nabla f\left(\Phi_{\lambda}+t^{\prime}\left(x-\Phi_{\lambda}\right)\right) d t^{\prime} d \lambda
\end{aligned}
$$

where first we have extended the integral over $t$ to the whole real line because $\int_{-\infty}^{0} f\left(\Phi_{\lambda}+t \theta\right) d t=0$ since the trajectory does not intersect $E_{a, b}$ which contains the support of $f$. The symbol $\Phi_{\lambda}^{\prime}$ means $(\partial / \partial \lambda) \Phi_{\lambda}$. In the last line, we have used the substitution of variables $t=t^{\prime}\left\|x-\Phi_{\lambda}\right\|$.
We now perform another change of variables $t^{\prime}=1-t$, followed by straight-forward manipulations, to obtain

$$
\begin{aligned}
b_{1,2}(x) & =\frac{1}{\pi} \int_{\lambda_{1}}^{\lambda_{2}} \int_{-\infty}^{\infty} \Phi_{\lambda}^{\prime} \cdot \nabla f\left(x+t\left(\Phi_{\lambda}-x\right)\right) d t d \lambda \\
& =\int_{-\infty}^{\infty} \frac{1}{\pi} \int_{\lambda_{1}}^{\lambda_{2}} \Phi_{\lambda}^{\prime} \cdot \nabla f\left(x+t\left(\Phi_{\lambda}-x\right)\right) d \lambda d t \\
& =\int_{-\infty}^{\infty} \frac{1}{\pi} \int_{\lambda_{1}}^{\lambda_{2}} \frac{1}{t} \frac{\partial}{\partial \lambda} f\left(x+t\left(\Phi_{\lambda}-x\right)\right) d \lambda d t \\
& =\int_{-\infty}^{\infty} \frac{1}{\pi t}\left[f\left(x+t\left(\Phi_{\lambda}-x\right)\right)\right]_{\lambda_{1}}^{\lambda_{2}} d t \\
& =\int_{-\infty}^{\infty} \frac{f\left(x-t\left(x-\Phi_{\lambda_{2}}\right)\right)}{\pi t} d t \\
& -\int_{-\infty}^{\infty} \frac{f\left(x-t\left(x-\Phi_{\lambda_{1}}\right)\right)}{\pi t} d t \\
= & H_{x-\Phi_{\lambda_{2}}} f(x)-H_{x-\Phi_{\lambda_{2}}} f(x) \\
= & H_{\theta_{2}} f(x)-H_{\theta_{1}} f(x)
\end{aligned}
$$

where $\theta_{i}$ was defined as $\theta_{i}=\left(\Phi_{\lambda_{i}}-x\right) /\left\|\Phi_{\lambda_{i}}-x\right\|$, for $i=1,2$.

\section{ACKNOWLEDGMENT}

We thank Dr. R.G. Wells of the Ottawa Heart Institute for supplying the parameters of the pie resolution phantom ("microdeluxe" phantom), which we scaled for our purposes.

\section{REFERENCES}

[1] P.-E. Danielsson, P. Edholm, J. Eriksson, and M. Magnusson Seger. "Towards exact 3D-reconstruction for helical cone-beam scanning of long objects. A new detector arrangement and a new completeness condition." Proc 1997 Meeting Fully 3D Image Reconstruction in Radiology and Nuclear Medicine D.W. Townsend and P.E. Kinahan, Eds. Pittsburgh, PA, 1997, pp.141-144.

[2] K.C. Tam, S. Samarasekera, and F. Sauer. "Exact cone beam CT with a spiral scan." Phys. Med. Biol. 43, 1015-1024, 1998.

[3] H. Kudo, F. Noo, and M. Defrise. "Cone-beam filtered backprojection algoirthm for truncated helical data. " Phys. Med. Biol. 43, 2885-2989, 1998.

[4] M. Defrise, F. Noo, and H. Kudo. "A solution to the long-object problem in helical cone-beam tomography." Phys. Med. Biol. 45, 623-643, 2000.

[5] H. Kudo, F. Noo, and M. Defrise. "Quasi-exact filtered backprojection algorithm for long-object problem in helical cone-beam tomography" IEEE Trans. Med. Imag. 19, 902-921, 2000.

[6] S. Schaller, F. Noo, F. Sauer, K. C. Tam, G. Lauritsch, and T. Flohr "Exact Radon rebinning algorithm for the long object problem in helical cone-beam CT" IEEE Trans. Med. Imag. 19, 361-375, 2000.

[7] A. Katsevitch. "Theoretically exact filtered backprojection-type inversion algorithm for spiral CT." SIAM J. Appl. Math. 62, 2012-2026, 2002.

[8] Y. Zou and X. Pan. "Exact image reconstruction on PI-lines from minimum data in helical cone-beam CT." Phys. Med. Biol. 49, 941959, 2004.

[9] R. Clackdoyle and M. Defrise. "Tomographic reconstruction in the 21st century. Region-of-interest reconstruction from incomplete data." IEEE Sig. Proc. Mag. 27, 60-80, 2010.

[10] F. Noo, R. Clackdoyle, and J. Pack. "A two-step Hilbert transform method for 2D image reconstruction." Phys. Med. Biol. 49, 3903-3923, 2004.

[11] Y. Zou, X, Pan, and E. Y. Sidky. "Image reconstruction in regions-ofinterest from truncated projections in a reduced fan-beam scan." Phys. Med. Biol. 50, 13-27, 2005.

[12] J. Pack, F. Noo, and R. Clackdodyle. "Cone-beam reconstruction using the backprojection of locally filtered projections." IEEE Trans. Med. Imag. 24, 70-85, 2005. 
[13] Y. Zou, X. Pan, D. Xia, and G. Wang. "PI-line-based image reconstruction in helical cone-beam computed tomography with a variable pitch." Med. Phys. 32, 2639-2648, 2005.

[14] Y. Zou, X, Pan, and E. Y. Sidky. "Theory and algorithms for image reconstruction on chords and within regions-of-interest." J. Opt. Soc. Am. A 22, 2372-2384, 2005.

[15] M. Defrise, F. Noo, R. Clackdoyle, and H. Kudo. "Truncated Hilbert transform and image reconstruction from limited tomographic data." Inv. Prob. 22, 1037-1053, 2006.

[16] S.G. Mikhlin. Integral Equations and Their Applications to Certain Problems in Mechanics, Mathematical Physics and Technology (New York: Pergamon), 1957.

[17] R. Alaifari and A. Katsevich. "Spectral analysis of the truncated Hilbert transform with overlap." SIAM J. Math. Anal. 46, 192-213, 2014.

[18] R. Alaifari, M. Defrise, and A. Katsevich. "Stability estimates for the regularized inversion of the truncated Hilbert transform." SIAM J. Math. Anal. 47, 797-824, 2015.

[19] R. Alaifari, M. Defrise, and A. Katsevich. "Asymptotic analysis of the SVD for the truncated Hilbert transform with overlap." Inverse Problems 32, 065005 (17pp), 2016.

[20] H. Kudo, M. Courdurier, F. Noo, and M. Defrise. "Tiny a priori knowledge solves the interior problem in computed tomography." Phys. Med. Biol. 53, 2207-2231, 2008.

[21] J. Pack and F. Noo. "Cone-beam reconstruction using 1D filtering along the projection of M-lines." Inv. Probs. 21, 1105-1120, 2005.

[22] Y. Ye, J. Zhu, and G. Wang. "Minimum detection windows, PI-line existence and uniqueness for helical cone-beam scanning of variable pitch.” Med. Phys. 31, 566-572, 2004.

[23] M. Kachelriess, M. Knaup, C. Penssel, and W. Kalender. "Flying focal spot (FFS) in cone-beam CT." IEEE Trans. Nucl. Sci. 53, 1238-1247, 2006.

[24] H. Tuy. "An inversion formula for cone-beam reconstruction." SIAM J. Appl. Math. 43, 546-552, 1983.

[25] S. Rit, M. Vila Oliva, S. Brousmiche, R. Labarbe, D. Sarrut, and G. Sharp. "The Reconstruction Toolkit (RTK), an open-source conebeam CT reconstruction toolkit based on the Insight Toolkit (ITK)" J. Phys.: Conf. Ser. 489, 012079, 2014.

[26] C. McCollough. Mayo Clinic. AAPM. NIH EB017095. EB017185 (NIBIB). 2015.

[27] H. Schoendube, K. Stierstorfer, and F. Noo. "Accurate helical conebeam CT reconstruction with redundant data" Phys. Med. Biol. 54, 4625-4644, 2009.

[28] E. A. Rashed, H. Kudo, and F. Noo. "Iterative region-of-interest reconstruction from truncated $\mathrm{CT}$ projection data under blind object support" Med. Imag. Techno. 27, 321-331, 2009. 